\section{Analysis of Motor-Vehicle Accidents and Blood Alcohol Concentration (BAC)}

\author{
Brenda L. Wainscots, MSII, *Jay L. Kovar, MD, \\ Linda D. Larsen, MPH \\ Department of Emergency Medicine, The University of \\ Texas-Houston Medical School, Houston, Texas
}

Purpose: To analyze the association of traffic accidents and fatalities with driver blood alcohol content (BAC) levels at or below legal limits in Texas $(0.10 \mathrm{~g} / \mathrm{dl})$.

Methods: A retrospective study of motor-vehicle accidents (MVAs) reported to the Texas Department of Public Safety involving injury or property damage greater than [US] $\$ 500$ and occurring on a public roadway. Accidents and fatalities were categorized according to driver BAC level.

Results: The 6,788 MVAs involved 10,664 drivers with BACs ranging from 0.00 to $0.20 \mathrm{~g} / \mathrm{dl}$. In $7,136(67 \%)$ cases, driver BAC was known. Of the 7,136 drivers, 6,400 $(90 \%)$ had a positive $\mathrm{BAC}$ test result $(\mathrm{BAC} \geq 0.01)$. Onethousand-four $(1,004)$ drivers $(16 \%)$ had a BAC under the legal limit in Texas. Thirty percent $(30 \%)$ of alcoholrelated fatalities involved a driver BAC below $0.10 \mathrm{~g} / \mathrm{dl}$. Poisson regression was used to predict the mean number of accidents and fatalities based on driver BAC. A decrease in BAC from 0.10 to $0.08 \mathrm{~g} / \mathrm{dl}$ represents a potential reduction in accidents by $23 \%$ and fatalities by $8 \%$.

Conclusions: The incidence of traffic accidents and fatalities in Texas with driver BACs below $0.10 \mathrm{~g} / \mathrm{dl}$ supports a reduction in current legal limits.
Information from Paramedic Injury Severity Assessment Can Aid Trauma Triage

*Erik Simmons, MS, ${ }^{1}$ Jerris $R$. Hedges, $M D, M S,{ }^{2}$ Lisa Irwin, RN, MPA/HA, ${ }^{1}$ Wilhelmine Maassberg, $R^{2}$

1. Oregon Health Division, Portland, Oregon

2. Oregon Health Sciences University, Department of Emergency Medicine, Portland, Oregon

Objective: To compare information contained in standard out-of-hospital trauma triage criteria vs. standard criteria plus paramedic estimate of severity of injury for determination of patient need for a trauma center.

Methods: A prospective, observational cohort analysis of trauma triage was performed using a stratified statewide sample of injured patients. Standard triage data (physiological parameters, anatomic injury, mechanism of injury, and co-morbid factors) were collected from EMS providers. The providers also ranked their assessment of patient injury severity ( 1 = minor, 2 = acute; nonlifethreatening; 3 = life-threatening; or 4 = requiring $C P R$ ). A patient was considered to need a trauma center if requiring major surgery within six hours of arrival, needing ICU admission, dying in hospital, or having an ISS $>16$. The relative triage information of standard criteria versus standard criteria plus provider assessment of injury severity were determined using logistic regression and receiver operating characteristic (ROC) curves.

Results: Three-hundred-six/1,063 $(28.8 \%)$ patients required a trauma center. Using a logistic regression model, the following standard triage parameters remained statistically associated $(p<0.05)$ with need for trauma center after inclusion of provider assessment of severity: 1) systolic blood pressure $<90$ torr; 2) respiratory rate $(<10$ or $>29 / \mathrm{min}), 3)$ Glasgow Coma Scale score $<13$; 4) penetrating injury (mid-thigh to head); 5) two or more obvious proximal long-bone fractures; 6) fall $>20$ feet). Severity ranks 3 and 4 had the greatest odds ratios (20:1 and $82: 1$, respectively). The model ROC areas were 0.88 with provider assessment of severity versus 0.83 without $(p<0.0001)$.

Conclusions: Standard triage criteria can benefit from inclusions of EMS provider clinical severity assessment. 